\title{
Quality Implication for Prognoses Success in Web Applications
}

\author{
Ruqia Bibi \\ University of Engineering and Technology, Taxila, 47080, Pakistan \\ E-mail: ruqia.kibria@yahoo.com \\ Munazza Jannisar and Mamoona Inayet \\ ${ }^{2}$ University of Engineering and Technology, Taxila, 47080, Pakistan \\ ${ }^{3}$ Fatima Jinnah Women University, Rawalpindi, 46000 Pakistan \\ E-mail: munazzajannisar@yahoo.com,moonainayet@gmail.com
}

\begin{abstract}
Massive increase in usage of World Wide Web makes it affective in every domain of life. Billions of people from different corners of world use internet for diverse purposes on daily bases. Quality acts as important key factor for web applications. Various methodologies have been proposed and many techniques have been deployed but, quality still lags behind. Our study infocuses a detailed survey based on methodologies, metrics and architecture that are used in modeling of web application. We carried out analysis and comparisons between techniques and evaluate major quality characteristics. Study at conclusion illustrates that beside functional requirements, non-functional requirements are also essential and acts as backbone for quality in web based applications.
\end{abstract}

Index Terms-Software Development Life Cycle (SDLC), Object-Oriented Techniques (OOT), Quality Assurance (QA), Object Oriented Design Methodology (OODM).

\section{INTRODUCTION}

The evolution of World Wide Web, Internet, Intranets, and Extranets had a significant impact on commerce, banking, finance, education and entertainment [15]. The web not only changed the way people communicate with each other but also change people's perspective. Billions of people are using web to transform the way they conduct their business that proved to be efficient and profitable in terms of money and time. The reason behind this adaptation is a cross functional platform of web based applications that allow large number of people to communicate having different underlying structure i.e. hardware, software, network connections etc.

Online business has surpassed the traditional business since last few years. Conferring to a forecast, progress with such rate for coming years will increase electronic commerce gigantically and usage will be in trillions. Internet has become basic source of information now days. Daily about 55 million people surf the internet for various purposes. Online traffic analyzers report double amount of users every 100 days. Such enormous usage of technology is fascinating but on the other side meeting with the challenges, quality assurance and customer satisfaction are also key issues.

In recent years, millions of people are using web for one or the other purpose and this number now has reached to its highest level. With every passing time user expectations for applications that require less development time and must be of top quality are increasing. On the other hand technical complexities of websites are making quality control and testing much more complex and subtle [24]. Thus automated testing can both be a challenge and an opportunity.

Estimating a web based application in terms of time and difficulty is a cumbersome task for web designers because of its unique domain as compared to applications of other domains. Web based services are gaining immense response from users which require companies or web designers to provide efficient and reliable services in terms of time and money. The problem faced by most of the designers is to correctly estimate the development effort in fixed time frame. The estimation models that have been used in traditional software development were not accurate for estimating effort of web based software development [13].

Before starting the Quality embedment, first step is to define what does an organization actually mean by it. Too often organizations operate on loose ends and ignore the flaws that should be operated to ensure quality [1]. Poorly developed web applications lead to high probability rate of failures.

Traditional analysis and design techniques such as Object-Oriented Techniques (OOT) and Structured Analysis and Design (SADT) couldn't provide acceptable solutions to avoid web crisis [14], thus highlights the need of new tools and disciplined approaches for development, deployment, implementation and maintenance of web based applications. Generic service attributes like throughput, security, reliability and maintainability were given more importance than domain related attributes.

Rest of paper is organized as; Section 2 discusses quality and development methodologies used for web based applications. Through analysis on mentioned 
techniques is presented in Section 3. A thorough analysis of the reported techniques is presented in Section 3. Section 4 conducts the conclusion of paper.

\section{LITERATURE REVIEW}

For determining quality of web based applications, non-functional requirements along with functional requirements contribute enormously. Inspecting quality without considering non-functional requirements not only result in customer dissatisfaction but also make the system or web based applications non-comply with desired standards. Based on above fact researcher [7] has proposed a model using GQM(Goal -Question- Metric) for deriving quality metrics and evaluating those derived metrics to rank a web based system. Multi element component comparison analysis technique has been used to rank a system.

Jyh et al [5] studies Chinese websites according to ALEXA'S rankings. Alexa collects data rendering to browsing history and global traffic, according to website's performance and daily usage rankings are assigned. Electronic commerce wide exposure has reached a point where it's considered a viable alternative for traditional commerce. Charismatic marketing strategies attract many customers daily thus increasing the e-commerce more than ever. If the business needs to use the internet as a marketing tool, essentials point is to focus on the services that can summon more and more user's traffic on the website. SERVQUAL model provides quality measurement framework for web based service. Multiple correspondence analysis (MCA), helps to develop coding sheet that explains marketing functions of websites is evenly distributed in five dimensions of service quality. Logo function is used in majority of websites 69 out of 70 showing firms are eager to out their business online. Study does not take user's perception of quality, in fact on the marketing strategy and web designer's effort to develop right web service for users. Internet follows bidirectional multiple communications, hence the measurement of quality in web based applications is different from any other customer services.

As the network environment is progressing every day, same is the need for end to end quality transfer. To make web services operative, service quality must be guaranteed. Service Level Agreement is an agreement between service providers and its users on promised facilities, which are needed by the users. Quality acts as back bone to glue the relationship for both users and providers. However, majority web servers do not provide beneficial strategies to enforce quality into process. Authors [6] furthermore, accentuated that a particular standard setup should be established for languages used in defining information on the web services quality, evaluation factors and the evaluations for themselves. In order to bring distinguished web services throughout the web server, research suggests a method that assigns priority based on these quality factors to web service message processing processes.
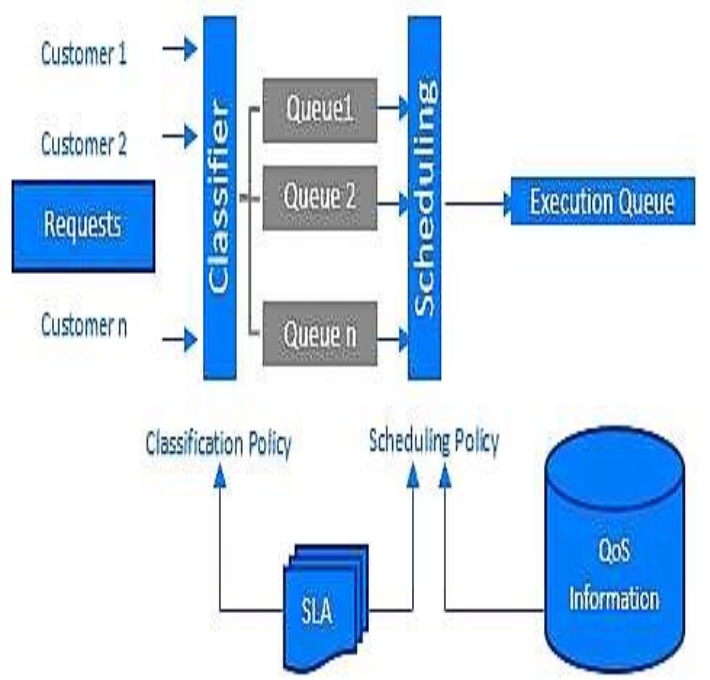

Fig.1. Model for Differentiated Web Server [6]

Method works dynamically, assign priorities to satisfy the quality factor for service level agreement and guarantee to have quality factor in as much as possible. Research did not mention compatible network speed attribute needed by differentiated services.

Domain-related attributes hold importance in selection and assessment of user's web services. Gaoyong and Huiling [3] in study put forward a model based on ontology and API hook, assessing the quality of web services of e-commerce websites and analyses these domain related service features. Authors mentioned in study that most of the QA techniques primarily focus on generic service attributes like accessibility, reliability, throughput, security, maintainability and latency; while overlooking the domain related attributes. These attributes narrate business contents of specific areas and service providers, from which customers determine quality of a service and its selection criteria. Model increases the accuracy rate of service evaluation, mechanism proposed put forward a strategy though which evaluation factors are adapted automatically to the needs of different service evaluations in different fields.

User needs for resources and user satisfaction, both terms effect resources in demand. Authors [11] emphasized service computing issues confronted by the web services. To fulfil required needs, users demand progressively for resources. From user's requirements credibility of resources, response time and network resources are analyzed, ultimately to achieve user's satisfaction. The service structure comprises of 3 roles (service providers, agents and services requestor) and three operations (publishing, discovery and binding). User of these resources from the service computing to conduct research work and the trust relationship need, confidence in mathematical model of resources by user, strategies of gain trust ultimately produce user satisfaction with services resources measure. Second step is to find the users credibility, done by research computing services to users. User's reliance on a resource is credibility of resource that reflects their dependencies in the service process which is main practice of evaluation of services. 
Quality assurance testing is methodical pattern of actions that ensure the conformance between website client and server products on an established set of measurements. Authors used six web testing tools to assess quality assurance criteria based on mentioned attributes. Evaluation of tools is done based on: structural quality, accuracy, timelines, response time, performance, latency and consistency. Testing is a process of checking software with intent of finding errors. Web based testing is searching for defect that can create erroneous performance on either client side or server side. Tools used for conducting this analysis are Doctor HTML, web performance trainer 2.0, rational site check, Net mechanic HTML toolbox and Webart. Research shows Rational site check is briefing QA set of tool that covers all of the quality assurance measures. For e-commerce domain this tool is not expensive and worth the investment. Rest of these tools does have similar functionalities but lack background of the all-encompassing the rational software unified development process. Rest of tools does have similar functionalities, except for extensive background knowledge of rational software unified development process. Person not familiar with quality assurance methodologies like students and beginners' web developers; free tools should. This can help supporting quality of their websites. Helm [4] suggested that these cited tools can be helpful at any level of understanding to carry out quality assurance activities of websites.

Due to immense increase in usage of Geographic Information Systems (GIS) and web services, software industry has witnessed exponential growth. Samuel et al [10] have proposed an algorithm to integrate GIS and web services in scenarios which require quick response to prevent failure or hazards. On request various services can be tailor for users. Proposed algorithm has been tested using different weights of preferences and requested user services.

Diverse research has been carried out in the field of quality proposing quality models and metrics, each with its own set of characteristics. These aspects mostly are not geared to focus on automatic collection \& classification in quality. Authors [9] made a preliminary effort and embedded the attribute i.e. automatic in websites for quality evaluation by putting forward an approach. Model proposed enlightens the automatable website quality evaluation. Almost 60 quality metrics are grouped for websites according to the ISO9126 Standard. Feasibility of automatic collection and classification in websites is shown in an empirical study that analyses different software metrics that depend on application domain. In order to generate automatic classification in websites, every quality attributes needs to be in terms of collectable quality metric. Principal Component analysis approach is applied on the dataset; size of data is not reduced to a lower dimension. Study also focuses on the application domain; analysis also shows if application domain affects the values of quality metrics being proposed. Three different domains are selected for this purpose: Banks, Airlines and Banks. Results conclude that; from the pool of quality metrics, half of the metrics are domain dependent.

In order to ensure quality of a system, semantic similarities must be checked between inputs and outputs of connected modules. Considering above, a new balanced approach has been proposed by researchers [8] which incorporate QoS (quality of service) and semantic quality and use them to present ranking and optimization criteria. The proposed approach has been tested for advantages and weaknesses by using a set of simulation experiments.

While discoursing about evolution in web field, gigantic increase in web applications has led to rapid software development; ignoring quality. A very little knowledge and techniques are applied to ensure key quality attributes. Offutt [2] highlights the need of all quality attributes, unique technological aspects and how the attributes can be implied to increase major factors. Applications now days have compelling needs including reliability, performance, maintainability, scalability, availability, security and usability quality attributes all at once. Table 1 shown below narrates quality parameters and the techniques. A web application depends on various other factors too beside the key attributes. Software applications have many aspects that implicate multiplicity. Paper uses term diversity to accentuate that producing web applications requires different kind of diversity. Web applications encompass distributed software that's developed using different languages, styles, might incorporate third-party components and software components in it interact with databases, users which bring diversity in it. Web software applications constitutes of large teams with multiple talents, experience and knowledge, including requirements engineer programmers, usability engineers, graphics designer, data base administrator, network experts and information layout specialists

Traditional structured design methodologies and object oriented techniques proved inadequate for examining implementation and testing phases of web based applications. For efficient and reliable web based systems, design and measurement should be given much insight because these aspects will highly reflect the performance of a system [14]. Researchers suggested some quality metrics for inspecting design, considering OODM (Object Oriented Design Methodology). Metrics include Robustness, Maintenance, Understandability, Adaptability, Coupling, Abstraction, Cohesion, Polymorphism, Modularity, Inheritance, and Reusability. All these were used to represent attributes of an entity. Above listed metrics will not only help in inspecting and making important decisions in the design phase but will save development cost and effort as well. The researchers concluded that model evaluating metrics can be used to compare different aspects of designs and OODM can be used for semantic web applications in future.

Over the last decade, the evolution of internet has marked as a success factor for many enterprises which were using traditional business model/ processes to carry out day to day activities. Moving to web based services help enterprises in expanding the business and capturing 
the customers. Developing web based systems without any definite plan would become a bottleneck which grows more complex if poorly handled and will circulate broad based problems across other systems. Comparatively web based crisis could be much worse than software crises. To avoid such catastrophic situation; one should follow wellplanned approach and advance tools for development, web implementation, deployment and maintenance. Such approaches must consider 1) specifications and unique features of medium 2) scenarios and increase in user profiles 3) skills and knowledge of developers 4) functioning environment. Research [15] elaborates principle and roles of web engineering, argued the need of web engineering approaches for the development of large scale, scalable and high quality web based systems. Comparison of developing traditional software systems and web based systems has also been assessed.

Several frameworks have been available to determine quality attributes of web services architecture. The researchers [16] developed quality attributes by using AQF (Architecture Quality Framework) to evaluate CORBA (Common Object Request Broker Architecture) framework and other common web services framework. The proposed framework served as a communication medium between user, problem dominion and conveying language. The framework is categorized into three groups- syntactic, semantic and pragmatic. Pragmatic is further classified into development and service -oriented framework. Service oriented describes non-functional properties that make up the system which encompasses quality of service and issues related to development of systems on the basis of architecture get handled in the development phase.

For testing web based applications a three tiered hierarchal strategy has been proposed [17]. The tiers were exhibited by flat operational profile, Markov chains and by structural testing models. It can be applied to selective components or functions to identify risks and ensure reliability. The proposed strategy provides integrated framework which would help in evaluating reliability and efficient testing of web based applications.

Along with reliability being the most important quality attribute, another aspect that needs to be given importance is security [18]. During the development phase, security modules should be incorporated in order to prevent application from getting failed. This will have a great impact on application's reliability. Application would be termed reliable if it's designed, implemented and tested considering reliability as benchmark.

With the advancement in technology and rapid increase in e-commerce, all retailing organizations are competing to attract customers thus highlighting the importance of CRM (customer relationship management) for gaining competitive advantage. The relationship among quality metrics, website quality and offline quality has been investigated [19]. Online features provided to the customers are termed as website quality and delivery services or after sale services are termed as offline quality. Websites are classified into three phases: pre, online and after sales. Pre includes advertisement of new products and services for attracting customers, improving the usability of interfaces for increasing purchase activities of customers coined as online and efficient delivering process termed as offline. Questionnaire analysis showed direct impact of satisfaction dimension on both trust and commitment dimension. The study suggests broader picture of quality by including offline quality and how customers regard the quality measures of e-retailing.

Researchers [20] point out the need of an automatic and objective quality evaluation of service definitions to assert the usability of the interface specifications. Integration of rich internet applications and web based services creating enriched front end interfaces raised the need. Optimized evaluation coverage requires help by domain experts in both quantitative and qualitative dimensions. Researchers analyzed related specifications against quality indicators by using graphical and statistical methods and emphasized on the availability of the results to developer, reviewer and user of web based specification to achieve quality of interoperability specifications.

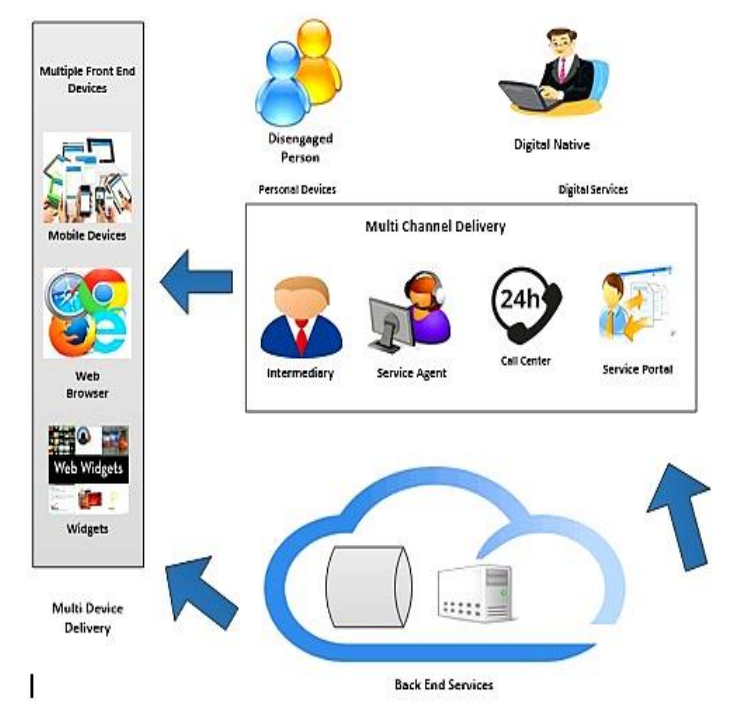

Fig.2. Back end Services Must Support Multiple Channel and Devices

Security has always been a concern for critical web based applications that require protection from malicious activities and vulnerabilities that aim to compromise user or organization information. To develop highly secure system, quality should be incorporated throughout SDLC (software development life cycle) phases [21]. This can only be achieved if each person in an organizational hierarchy properly understands software quality and measurable indicators to assess overall development quality. In depth security analysis should be done proactively so that security issues get discovered in initial stages thus reducing threats from external attackers.

Researchers [22] highlight the benefits of adopting agile engineering for web development over the traditional engineering methods. As compared to traditional methods, agile arrange the project tasks according to superiority in to small manageable modules. Agile development put emphasis on auditing and adopting which yields software that satisfies customer needs. To 
ensure compliance to customer needs, each finished module is cross checked for quality. This eliminates a scenario where customer felt dissatisfied and required a change that would not only cost much but will also waste time. Agile web development guarantees high quality and error free product because of intensive and thorough testing.

Table 1. Quality Parameters for Web Based Applications

\begin{tabular}{|c|l|c|}
\hline Quality Parameters & \multicolumn{1}{|c|}{ Meaning } & Possible Values \\
\hline Availability & $\begin{array}{l}\text { Website services are accessible for use } \\
24 / 7 / 365\end{array}$ & Yes, No \\
\hline Interoperability & $\begin{array}{l}\text { It's the working of Miscellaneous systems } \\
\text { together. It should be able to manage } \\
\text { concurrent processing of tasks. }\end{array}$ & Yes, No \\
\hline Maintainability & $\begin{array}{l}\text { Feasibility and level of ease with which } \\
\text { product's maintenance is carried out. }\end{array}$ & Yes, No \\
\hline Performance & Under certain load; how fast website works. & Yes, No \\
\hline Reusability & $\begin{array}{l}\text { Reusability is checking the software module for } \\
\text { reuse. With what ease it can be integrated into } \\
\text { new components. }\end{array}$ & $\begin{array}{l}\text { Websites must ensure its desirable functionality } \\
\text { over mentioned set of services without any } \\
\text { failure. }\end{array}$ \\
\hline Reliability & $\begin{array}{l}\text { It shows level of security integrated, protection } \\
\text { of personal data, and dealing with cybercrime } \\
\text { issues. }\end{array}$ & Yes, No \\
\hline Security & Website is user friendly and easy in use. & Yes, No \\
\hline
\end{tabular}

Table 2. Analysis of Quality Parameters for Web Based Applications

\begin{tabular}{|c|c|c|c|c|c|c|c|c|c|c|c|c|c|c|c|c|c|c|c|c|}
\hline $\begin{array}{c}\text { Quality } \\
\text { Parameters }\end{array}$ & {$[2]$} & [3] & [4] & {$[5]$} & [6] & [7] & [8] & [9] & [10] & [11] & [14] & [15] & [16] & [17] & [18] & [19] & [20] & [21] & [22] & $\begin{array}{c}\text { S.Zhou } \\
\text { and } \\
\text { X.Liu }\end{array}$ \\
\hline Availability & $\checkmark$ & $\checkmark$ & $\checkmark$ & & $\checkmark$ & $\checkmark$ & $\checkmark$ & $\checkmark$ & & & $\checkmark$ & $\checkmark$ & $\checkmark$ & $\checkmark$ & & $\checkmark$ & $\checkmark$ & $\checkmark$ & $\checkmark$ & $\checkmark$ \\
\hline Interoperability & & & $\checkmark$ & & & & & & $\checkmark$ & & & & & & $\checkmark$ & & $\checkmark$ & & $\checkmark$ & \\
\hline Maintainability & $\checkmark$ & $\checkmark$ & & & $\checkmark$ & $\checkmark$ & & $\checkmark$ & & $\checkmark$ & & $\checkmark$ & $\checkmark$ & $\checkmark$ & & & $\checkmark$ & & $\checkmark$ & $\checkmark$ \\
\hline Performance & $\checkmark$ & & $\checkmark$ & $\checkmark$ & & $\checkmark$ & $\checkmark$ & $\checkmark$ & $\checkmark$ & & & $\checkmark$ & & & $\checkmark$ & $\checkmark$ & $\checkmark$ & & $\checkmark$ & \\
\hline Reusability & & & & $\checkmark$ & & & $\checkmark$ & & & & $\checkmark$ & & $\checkmark$ & $\checkmark$ & & & $\checkmark$ & & & $\checkmark$ \\
\hline Reliability & $\checkmark$ & $\checkmark$ & $\checkmark$ & $\checkmark$ & $\checkmark$ & $\checkmark$ & $\checkmark$ & $\checkmark$ & $\checkmark$ & $\checkmark$ & & $\checkmark$ & $\checkmark$ & $\checkmark$ & $\checkmark$ & $\checkmark$ & $\checkmark$ & $\checkmark$ & $\checkmark$ & $\checkmark$ \\
\hline Security & $\checkmark$ & & & & & $\checkmark$ & & $\checkmark$ & $\checkmark$ & $\checkmark$ & & & & $\checkmark$ & $\checkmark$ & & $\checkmark$ & $\checkmark$ & & $\checkmark$ \\
\hline Usability & $\checkmark$ & & & & $\checkmark$ & $\checkmark$ & & $\checkmark$ & & $\checkmark$ & & & & $\checkmark$ & & $\checkmark$ & $\checkmark$ & & & $\checkmark$ \\
\hline
\end{tabular}

\section{ANALYSIS}

Prognoses of quality factors debate about comprehensive research on quality factors and attributes. Quality requirements demand the key performance of factors such as performance, availability, security, reliability and scalability. For effective implementation of web based applications these factors mentioned above in essence provide considerable influence.

Development methodologies OODM, Agile and Reverse engineering are analyzed. Agile methodologies follow rapid application development that leads to less focus on quality factors and more on timely delivery. Major obstacle in producing successful web application is lack of communication with consumer. To sort out the issue, various providers have generated Website and offline quality to run these websites with a strategy known as institution-based relationship quality [19]. Different practices used to study the quality attributes are MAC, AQF, GQM, API, Ontology, QoS and Priority Allocation Method. Domain associated functionality is explicitly discussed by Rio and Abreu [8]. Results from the matrices describe that; about half of the quality attributes in web based applications are domain dependent.

Table 2 and table 3 depict the results of study of quality attributes defined in evaluation table 1. Our work emphasize on examination of eight parameters for website quality affecting factors.

Table 2 and table 3 shows that factors that play major part in success of quality based web applications are usability, security, reliability, performance and availability. Study and various approaches previously support the idea in table 2 and 3, showing the substantial importance of these factors in any web based application. In fact this cannot be considered as a final list on which success of web application can depend. Table 2 and 3 also reveal that all the techniques except [14] have mentioned reliability as a necessary attribute for web based application. Highly reliable software is a critical aspect for applications that rely on safety, such as telecommunications, medical devices-commerce and aerospace.

Usability happens to be one of the major attribute in web applications. As compared to traditional software apps where they have users, websites have customers [2]. 
Level of ease with which customer can use software, earns the customer's interest.

Along with other issues, security has always been a major concern for web applications. Developers are striving hard to assure CIA (Confidentiality, integrity, Availability). In spite of adapting security-oriented development, websites have been cracked and information has been compromised. All the cited research has considered techniques which merely analyze security in general but in [21] an in-depth analysis method has been proposed which aims to analyze security at each and every development step of web based applications.

The most crucial thing customer expects from web application is its $24 / 7$ availability. If a website faces down-time even of some minutes, customers will switch to other sites and that will cost much to the company. Customer dissatisfaction is something that any company would want to avoid as much as they can. All the researchers have considered availability as a success factor for web applications except [5, 10, and 18]. Another quality attribute that has been discussed in research is performance.

Nielsen [12] claims that availability of website $24 / 7 / 365$ is also essential to keep users concentration towards what you offer. Slight delay in content loading loses user's interest. New technological release in market fuel up new web applications demands [2]. After a website is launched; maintainability factor goes in parallel. Changes are gradually captured and done by software developers as soon as the website is put to work thus; maintainability also holds place of major requirement in web based applications. Service back end designing should be highly reusable and with well-defined interfaces. This is essential for interoperability and data exchange, as the front ends services are progressive and enriched by apps. Only Gottschick and Restel [20] talked about interoperability in their research study.
Interoperability does have an impact on the websites working and is equally essential for its working.

Table 4 describes evaluation factors for web based application that are highlighted in table 3 .

\section{SUGGESTIONS AND CONCLUSION}

Our work major focus remained on effect and significance of QUALITY for web based applications. Various approaches have been proposed to master the task of quality measurement in web applications, we have studied few methodologies. Quality parameters cited in this paper depict that only web applications had reasons to satisfy all the quality attributes. Technological advancements now days are at much faster rate than development. With such gigantic web usage, rapid progressive changes provide quality less chance to compete equally. Production of quality web based application demands incorporation of quality at each and every phase of software development life cycle. Internet is the second name for 24/7 used services. This demands quality as an essential feature to be engrafted in websites. Websites are expected to be operational throughout every day of year providing required services in a reliable way. These elite characteristic require concurrent availability and timely response. Eight parameters are debated for quality evaluation in web application; table 03. Each of these supports the point that quality should be in-focused in web application and has a significant effect on these parameters. In nuts shell, quality attributes provide origins for successful web application.

Future work for this survey paper includes the calculation of individual effect of each quality attribute on web applications and their measurement using metrics. Measurement of parameters on individual bases will help to differentiate the separate impact of each parameter.

Table 3. Evaluation Parameters for Web Based Applications

\begin{tabular}{|c|c|c|}
\hline Evaluation Parameters & Meaning & Possible Values \\
\hline Development & Development methods being used. & OODM, Agile Web Development \\
\hline Domain specific functionality & Domain dependent or not. & Yes, No \\
\hline Offline quality & Deployment and maintenance services. & Yes, No \\
\hline Performance & $\begin{array}{l}\text { System performance under different workload } \\
\text { scenarios. }\end{array}$ & Efficiency, response time, resource usage \\
\hline Reliability & Evaluating system in terms of reliability. & $\begin{array}{l}\text { Accuracy, failure rate, fault tolerant, } \\
\text { recoverability }\end{array}$ \\
\hline Security & $\begin{array}{c}\text { Ensuring web security by applying available } \\
\text { techniques. }\end{array}$ & Yes, No \\
\hline Testing & Applicable methods to test websites quality. & $\begin{array}{l}\text { 3-Tiered hierarchical strategy, tools used for } \\
\text { testing }\end{array}$ \\
\hline Website quality & Ways to evaluate quality of a website. & $\begin{array}{c}\text { GQM, MAC,AQF, Ontology, API, Priority } \\
\text { Allocation Method, QoS }\end{array}$ \\
\hline
\end{tabular}


Table 4. Evaluation Parameters for Web Based Applications

\begin{tabular}{|c|c|c|c|c|c|c|c|c|c|c|c|c|c|c|c|c|c|c|c|}
\hline 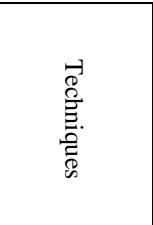 & 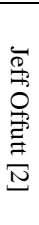 & 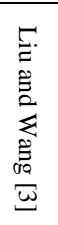 & 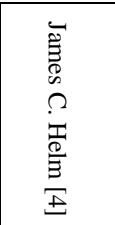 & 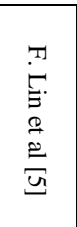 & 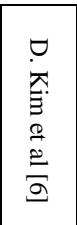 & 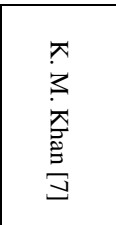 & 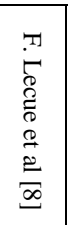 & $\begin{array}{l}D \\
20 \\
0 \\
0 \\
0 \\
\tilde{0} \\
0\end{array}$ & 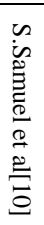 & 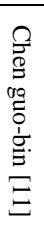 & 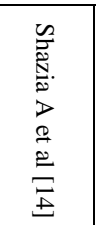 & 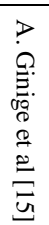 & 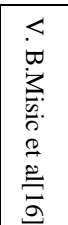 & 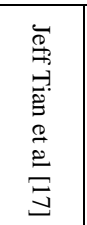 & 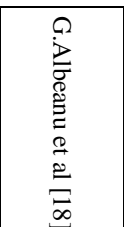 & 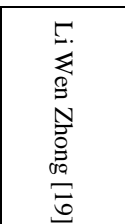 & 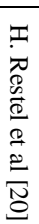 & 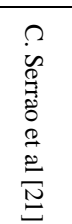 & 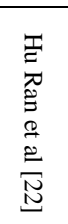 \\
\hline Development & & & & & & & & & $\checkmark$ & & OODM & $\checkmark$ & & & & & & & Agile \\
\hline $\begin{array}{c}\text { Domain } \\
\text { functionality }\end{array}$ & & $\checkmark$ & $\checkmark$ & & $\checkmark$ & & $\checkmark$ & $\checkmark$ & $\checkmark$ & & & $\checkmark$ & & & & & $\checkmark$ & $\checkmark$ & \\
\hline $\begin{array}{l}\text { Offline } \\
\text { Quality }\end{array}$ & & $\checkmark$ & & & & & & $\checkmark$ & & & & & & & & $\checkmark$ & & & \\
\hline Performance & $\checkmark$ & & $\begin{array}{c}\text { Response } \\
\text { time }\end{array}$ & $\checkmark$ & & $\begin{array}{c}\text { Response } \\
\text { time }\end{array}$ & $\checkmark$ & $\checkmark$ & $\checkmark$ & & & $\checkmark$ & & & Efficiency & Efficiency & $\checkmark$ & & $\checkmark$ \\
\hline Reliability & $\checkmark$ & $\checkmark$ & $\checkmark$ & $\checkmark$ & $\checkmark$ & $\begin{array}{c}\text { Failure } \\
\text { rate }\end{array}$ & $\checkmark$ & $\checkmark$ & $\checkmark$ & $\checkmark$ & & $\checkmark$ & $\checkmark$ & $\checkmark$ & $\checkmark$ & $\checkmark$ & $\checkmark$ & $\checkmark$ & $\checkmark$ \\
\hline Security & $\checkmark$ & & & & & $\checkmark$ & & $\checkmark$ & $\checkmark$ & $\checkmark$ & & & & $\checkmark$ & $\checkmark$ & & $\checkmark$ & $\begin{array}{c}\text { In- } \\
\text { depth }\end{array}$ & \\
\hline Testing & & & $\begin{array}{c}\text { Rational } \\
\text { Site } \\
\text { Check }\end{array}$ & & & & & & $\checkmark$ & & & & & \begin{tabular}{|c|}
$3-$ \\
tiered \\
strateg \\
$y$
\end{tabular} & $\checkmark$ & & & & \\
\hline $\begin{array}{l}\text { Website } \\
\text { Quality }\end{array}$ & $\checkmark$ & API & $\checkmark$ & MCA & PAM & GQM & QoS & $\checkmark$ & $\checkmark$ & $\checkmark$ & $\begin{array}{l}\text { Design } \\
\text { Quality } \\
\text { Metric }\end{array}$ & $\checkmark$ & $\overline{\mathrm{AQF}}$ & & & $\checkmark$ & $\checkmark$ & & $\checkmark$ \\
\hline
\end{tabular}

\section{REFERENCES}

[1] http://www.ibm.com/developerworks/rational/library/dec0 4/bessin/\#N1005F

[2] J. Offutt, "Web software application quality attributes," 2002.

[3] L. Gaoyong and W. Huiling, "A method for evaluating the service quality of E-commerce websites based on ontolog," 2008.

[4] J.C. Helm, "Web based application quality assurance testing," 2000.

[5] F. Lin, K. Huarng, Y. Chen and S. Ling, "Quality evaluation of web services," International conference on ecommerce technology, 2004.

[6] D. Kim, S. Lee, S. Han and A. Abraham, "Improving web services performance using priority allocation method," International conference on next generation web services, 2005.

[7] K.M. Khan, "Assessing quality of web based systems," IEEE, 2008.

[8] F. Lecue and N. Mehandjiev, "Seeking quality of web service composition in a semantic dimension," IEEE transaction on knowledge and data engineering, June 2011.

[9] A. Rio and F.B. Abreu, "Websites quality: Does It Depend on Application Domain." International conference on the quality of information and Communication technology, 2011.

[10] S.J. Samuel and T. Sasipraba, "A quality based web service composition algorithm for the integration of emergency web services," IEEE 2010.

[11] C. Guo-bin, "Research in customer satisfaction based on web services resources," International conference on network computing and information security, 2011.

[12] J. Nielsen, "Designing web usability," New Riders Publishing, Indianapolis, 2000.

[13] http:www.acadjournal.com/2008/v23/part6/p7
[14] Shazia, M.Shoaib, A.Shah and F.Majeed, "Software design quality metrics for web based applications," Pakistan Journal of science, March, 2011.

[15] S. Murugesan, Y. Deshpande, S. Hensen, A. Ginige, "Web Engineering: A new discipline for development of web based systems," pp.3-13, 2001.

[16] V.B. Misic and D. Ibrahim, "A quality evaluation framework for web service architecture," 2006.

[17] J. Tian, L. Ma, Z. Li and A.G. Koru, "A hierarchical strategy for testing web based applications and ensuring their reliability," COMPSAC, 2003.

[18] G. Albeanu, A. Averian and I. Duda, "Web software reliability engineering," December 2009.

[19] L.W. Zhong and J.A. Ying, "The impact of website and offline quality on relationship quality," IEEE, 2008.

[20] J. Gottschick and H. Restel, "An empirical evaluation of the quality of interoperability specifications for the web," in EUROMICRO conference on software engineering and advanced applications, 2010.

[21] N. Teodoro and C. Serrao, "Web application security: Improving critical web based applications quality through in depth security analysis," 2011.

[22] H. Ran, W. Zhuo and X. Jianfeng, "Web quality of agile web development," IITA International conference on services sciences and engineering, 2009.

[23] http://www.iwebsitetemplate.com/guides/webdesign/agile-web-development-and-its-benefits/

[24] E. Miller, "The Website Quality Challenge," 2008.

[25] Ahed Abugabah, "Measuring information quality and systems quality," International Journal of Information Technology and Computer Science, Vol 11, Issue 2, April 2015.

[26] Emad Elabd, "A Dynamic Reputation-Based Approach for Web Services Discovery," International Journal of Information Technology and Computer Science, Vol 7, No 8, July 2015. 


\section{Authors' Profiles}

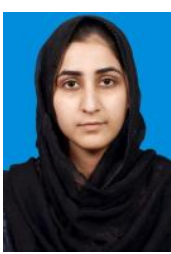

Ruqia Bibi is MS scholar in the Software Engineering Department at University of Engineering and Technology Taxila, Pakistan. She graduated from Fatima Jinnah Women University Rawalpindi, Pakistan in Software Engineering in July 2012. Her focused research areas are Mobile Application Development, Model Driven Engineering, Computer Networks and Digital Signal Processing.

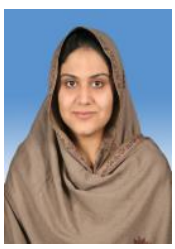

Munazza Jannisar is currently enrolled as MS scholar in the Software Engineering Department at University of Engineering and Technology Taxila, Pakistan. She graduated from University of Engineering and Technology Peshawar, Pakistan in Software Engineering in August 2012. She is currently research associate at Comsats Institue of Information and Technology Abbottabad.
Her focused research areas are Software Testing and Quality Assurance, Computer Networks, Artificial Intelligence, Data mining and Recommender Systems.

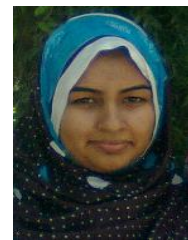

Mamoona Inayet has done Bachelors from Fatima Jinnah Women University Rawalpindi, Pakistan in Software Engineering in July 2012. Her research interests include Mobile Networks, Web Development, Quality Assurance and Requirement Engineering.

How to cite this paper: Ruqia Bibi, Munazza Jannisar, Mamoona Inayet,"Quality Implication for Prognoses Success in Web Applications", International Journal of Modern Education and Computer Science(IJMECS), Vol.8, No.3, pp.37-44, 2016.DOI: 10.5815/ijmecs.2016.03.05 